\title{
Survival and Predictors of Death for Patients with Broncho- pulmonary Carcinoid at a Danish Tertiary NET Centre
}

\author{
LINDA SKIBSTED KORNERUP, GITTE DAM and HENNING GRONBAEK \\ Department of Hepatology and Gastroenterology, Aarhus University Hospital ENETS Centre of Excellence, \\ Aarhus University Hospital, Aarhus, Denmark
}

\begin{abstract}
Background/Aim: Bronchopulmonary carcinoids comprise of typical carcioids (TC) and atypical carcinoids (AC). We present characteristics and associated mortality in patients with TC and AC followed-up at a Danish NET Centre between 2000-2014. Patients and Methods: Data were obtained from medical records, computed tomographic scans, and pathology reports. Mortality was investigated by KaplanMeier survival analysis. Results: We included 68 patients, 55 with TC (81\%) and 13 with AC (19\%). Median follow-up was 5.6 years (range $=1.0-26.3$ ). Fourteen patients died; 13 were smokers, and nine had a Ki67 index $>2 \%$. Metastatic disease, smoking and male gender were significantly associated with risk of dying within the first 5 years (log-rank, all $p<0.05)$, while Ki67 index $>2 \%$ showed a trend for being associated with risk of death $(p=0.11)$. Conclusion: Survival is strongly associated with tumor type and current/previous smoking. Metastatic disease at diagnosis and male gender are predictors of death within 5 years.
\end{abstract}

Bronchopulmonary neuroendocrine tumors (BP-NETs) account for $20-25 \%$ of all neuroendocrine tumors and 20 $25 \%$ of all lung cancer. BP-NETs comprise of typical carcinoids (TC), atypical carcinoids (AC), large-cell neuroendocrine carcinomas (LCNEC) and small-cell lung carcinomas (SCLC). Bronchial carcinoids (TC 80\%, AC $20 \%$ ) represent $1-2 \%$, LCNEC $<1 \%$, and SCLC $10 \%$ of all lung cancer (1-4). TCs are considered low-grade and ACs

This article is freely accessible online.

The Abstract of this article was presented as a poster at the 12th ENETS Conference in Barcelona, Spain, 11-13 March 2015 (21).

Correspondence to: Linda Skibsted Kornerup, MD, Department of Hepatology \& Gastroenterology, Aarhus University Hospital, Nørrebrogade 44, 8000 Aarhus C, Denmark. E-mail: lindajen@rm.dk

Key Words: Neuroendocrine tumors, bronchial neuroendocrine tumors, carcinoid, typical, atypical, Ki67 index. intermediate-grade NETs while LCNECs and SCLCs are high-grade. Survival rates for each subtype reflect these differences. Five-year survival in a retrospective study of 12,345 patients diagnosed with BP-NET between 1978-1997 was $87 \%$ for TC, $44 \%$ for AC, $15 \%$ for LCNEC, and $2 \%$ in SCLC (5). However, other studies described 5-year survival of $87-100 \%, 61-88 \%, 15-57 \%$ and $<5 \%$ for TC, AC, LCNEC and SCLC, respectively (1-3).

Studies on cytogenetics found distinct chromosomal imbalances in BP-NET but to a lesser degree than SCLC. Genetic alterations were less widespread and involved narrower gene regions in BP-NET compared to SCLC, but several alterations were identical for both BP-NET and $\operatorname{SCLC}(6,7)$.

Studies investigating an association between smoking and development of AC and TC are conflicting. Several studies concluded that smoking was not associated with bronchial carcinoid development, whereas two other studies found higher smoker prevalence among AC patients compared to healthy individuals or patients with TC (8-11). An association between smoking status and survival has not been demonstrated $(3,9,12,13)$. Other predictors of mortality are histological type and stage at diagnosis $(3,9)$, whereas female gender was a negative predictor in one study (3).

The aim of the present study was to describe demographic, immunohistochemical, and biochemical characteristics, survival, and predictors of death for all patients diagnosed with bronchial carcinoid and referred to our ENETS Centre of Excellence at Department of Hepatology and Gastroenterology, Aarhus University Hospital, Denmark in the period 2000-2014.

\section{Patients and Methods}

This is a retrospective cohort study. All patients from Western Denmark diagnosed with TC and AC followed at the ENETS Centre of Excellence at Aarhus University Hospital, Denmark, between 2000-2014 were included. According to the Danish "Act on Research Ethics Review of Health Research Projects", act number 593 of 14 July 2011 , section 14,1 only health research studies are notified to the Committees. The Committees did not consider this study to be a health research study (section 2,1) (Ethics journal no: 179/2015). 
Patients were evaluated in a multidisciplinary setting. All patients were hospitalized for disease staging and had an octreotide scintigraphy or $\mathrm{Ga}^{68}$ - 1,4,7,10-tetra-azacyclododecane tetra-acetic acid 1-Nal3-octreotide (DOTANOC) positronemission tomography (PET)-computed tomographic (CT) scan performed. Our multidisciplinary team decided if patients were suitable for surgery, radiofrequency ablation, chemoembolization, medical treatment, chemotherapy, stereotactic radiotherapy, peptide receptor radionuclide treatment, or other experimental treatments. Patients were followed-up at our Outpatient Clinic after 1 month and subsequently every 3-6 months during follow-up. CT scan and plasma chromogranin A $(\mathrm{CgA})$ determination preceded every visit. Plasma $\mathrm{CgA}$ was measured by a specific assay at Rigshospitalet, Copenhagen, Denmark as previously described (14).

Demographic, clinical, biochemical, and immunohistochemical data were obtained. TNM classification, histopathological (TC and AC) and immunohistochemical $[\mathrm{CgA}$, synaptophysin, CD56, tyrosine transcription factor-1 (TFF-1), and Ki67-index] data were examined based on pathology reports retrieved from a national Danish pathology database. An experienced pathologist with expertise in neuroendocrine tumors evaluated all biopsies.

Demographic and clinical features. We examined the following demographic and clinical features: patient age at diagnosis, gender, smoking status at diagnosis, primary resections, somatostatin receptor imaging ( $\mathrm{In}^{111}$ pentetreotide scintigraphy (SPECT) or $\mathrm{Ga}^{68}$ DOTANOC PET) combined with CT scans, and plasma CgA. All data were obtained from patients' medical records. Smoking status was divided into non-smokers, previous smokers (more than 6 months since quitting), and current smokers (ongoing smoking and $<6$ months since quitting).

Pathological features. Histopathological diagnosis was established by a specialized pathologist employing light microscopy according to The 2004 WHO Classification of Lung Tumors (15). TC was defined as a tumor $\geq 0.5 \mathrm{~cm}$ with carcinoid morphology, $<2$ mitoses per $2 \mathrm{~mm}^{2}$ [10 high-power fields (HPF)], and lack of necrosis. AC was defined as a tumor with carcinoid morphology and 2 to $<10$ mitoses per $2 \mathrm{~mm}^{2}$ or necrosis. Ki67 index, a marker of proliferating cells, was estimated according to the most agreedupon procedure (16). TNM classification was determined according to the seventh edition of the TNM Staging for Lung Cancer (17) based on data obtained from pathology reports (from biopsies or resections from primary surgery), medical records, and CT scan reports.

Statistics and survival analysis. The chi-square test and twosample Wilcoxon test were used to test the distribution of binomial and continuous data as appropriate. Categorical data are shown as absolute numbers (valid cases, percentages). Continuous variables are presented as medians with range unless otherwise noted. To investigate association between specific risk factors, we calculated total mortality and constructed unadjusted KaplanMeier curves. Log-rank statistics was used to test associations. Tests were considered statistically significant when $p$-values were less than 0.05 (two-sided test). Statistical analyses were performed using Intercooled STATA version 11 (StataCorp LP, College Station, TX, USA).

\section{Results}

Demographic, clinical and biochemical features. Demographic, clinical and biochemical features are summarized in Table I. We included 68 patients, 24 men (35\%) and 44 women (65\%). Median follow-up was 5.6 years (range $=1.0-26.3$ years). The median age at diagnosis was 61 (range=6-79 years) with no difference between men and women [56 (range=32-76) vs. 62 (range=6-79) years, respectively, $(p=0.28)$. The median age was significantly higher for patients with AC compared to patients with TC. Seventy-eight percent $(n=53)$ of the study population underwent primary surgery, while $22 \%(n=15)$ had advanced disease or significant comorbidity at diagnosis contradicting surgery. We found no difference in primary surgery rates by tumor type or by gender. Thirty percent $(n=19)$ had a positive somatostatin receptor imaging at the first hospitalization at our department for disease staging. This was not associated with death, tumor type, gender, smoking status, or metastatic disease at diagnosis.

The median CgA level was not significantly different in patients with TC from that in patients with AC (92.5 pmol/1 vs. $153 \mathrm{pmol} / \mathrm{l} ; p=0.44)$.

Smoking status. The frequency of current or former smokers and gender distribution of smokers was similar in with TC and $\mathrm{AC}$ groups $(p=0.54)$. Current/former smokers were, however, overall significantly older than non-smokers (log rank, $p=0.016$ ).

Pathological features. The study population was subdivided according to tumor type. Fifty-five patients (81\%) were diagnosed with TC and 13 (19\%) with AC. There was a nonsignificant distribution of women between TC and adenocarcinoma groups; the female to male ratio in those with TC was 1.6:1, whereas that for $\mathrm{AC}$ was 3.3:1. The median $\mathrm{Ki} 67$ index was significantly lower at $2 \%$ in patients with TC than that in patients AC $(10 \% ; p<0.0001)$.

In our cohort, $64 \%(n=35)$ had T1 disease, $27 \%(n=15)$ T2 disease and $9 \%(n=5)$ T3 disease at diagnosis. There was no difference in T-class distribution between TC and AC, but we observed a higher T-class in men compared with women $(p=0.01)$.

Seventy-one per cent of our study population had nonmetastatic disease (N0, M0), 13\% N1, 13\% N2 and 3\% N3 disease at diagnosis. There was a significant difference in $\mathrm{N}$ class distribution between TC and $\mathrm{AC}(p=0.02)$ with a larger fraction of patients with $\mathrm{N}>1$ in $\mathrm{AC}$ than TC. We found no difference in gender distribution between $\mathrm{N}$-classes $(p=0.84)$. Twelve percent $(n=8)$ of all patients had distant metastases (M1a/M1b) at diagnosis. There was no significant difference in distribution of $\mathrm{M}$-classification between tumor types $(p=0.56)$ or genders $(p=0.14)$. 
Table I. Demographic, clinical, and biochemical description and TNM classification of patients with typical carcinoid (TC) and atypical carcinoid (AC).

\begin{tabular}{|c|c|c|c|}
\hline Characteristic & $\mathrm{TC}(\mathrm{N}=55)$ & $\mathrm{AC}(\mathrm{N}=13)$ & $p$-Value \\
\hline Gender, n (\%) & & & 0.31 \\
\hline Men & $21(38)$ & $3(23)$ & \\
\hline Women & $34(62)$ & $10(77)$ & \\
\hline Female:male ratio & $1.6: 1$ & $3.3: 1$ & \\
\hline Median age at diagnosis (range), years & $56(6-79)$ & $65(54-74)$ & 0.026 \\
\hline Men & $55(32-76)$ & $58(57-74)$ & 0.13 \\
\hline Women & $61(6-79)$ & $67(54-73)$ & 0.10 \\
\hline T-classification, $\mathrm{n}(\%)$ & & & 0.17 \\
\hline $\mathrm{T} 1$ & $28(67)$ & $7(54)$ & \\
\hline $\mathrm{T} 2$ & $10(24)$ & $5(38)$ & \\
\hline $\mathrm{T} 3$ & $4(9)$ & $1(8)$ & \\
\hline $\mathrm{N}$-classification, $\mathrm{n}(\%)$ & & & 0.020 \\
\hline No & $37(74)$ & $10(77)$ & \\
\hline N1 & $8(16)$ & 0 & \\
\hline $\mathrm{N} 2$ & $5(10)$ & $1(8)$ & \\
\hline $\mathrm{N} 3$ & 0 & $2(15)$ & \\
\hline M-classification, n (\%) & & & 0.61 \\
\hline M0 & $48(87)$ & $12(92)$ & \\
\hline M1 & $7(13)$ & $1(8)$ & \\
\hline Ki67 index, n (\%) & & & $<0.001$ \\
\hline$<2$ & $27(57)$ & 0 & \\
\hline $3-20$ & $20(43)$ & $13(93)$ & \\
\hline Median $\mathrm{pCgA}$ (range), pmol/1 & $92.5(27-7,600)$ & $153(44-20,500)$ & 0.44 \\
\hline Receptor scintigraphy/Ga-DOTANOC PET-CT, n (\%) & & & 0.56 \\
\hline Positive & $16(31)$ & $3(23)$ & \\
\hline Negative & $35(69)$ & $10(77)$ & \\
\hline Smoking status, n (\%) & & & 0.54 \\
\hline Non-smoker & $2(40)$ & $4(31)$ & \\
\hline Current/previous smoker & $33(60)$ & $9(69)$ & \\
\hline
\end{tabular}

Survival analysis. Fourteen patients died during follow-up $(21 \%)$. Twenty-nine percent $(n=7)$ of men died compared to $16 \%(n=7)$ of women. Sixteen percent $(n=9)$ of patients with TC died compared with $38 \%(n=5)$ of those with AC.

There was no significant survival difference between patients with TC and those with $\mathrm{AC}$ at 5-years follow-up $(p=0.49)$, while survival was significantly higher in the TC group compared with the AC group when analyzing the entire follow-up period ( $p=0.02)$ (Figure 1). Male gender was associated with higher risk of dying within 5 years $(p=0.04)$, however, there was no significant survival difference between men and women for the entire follow-up period ( $p=0.20$ ) (Figure 2).

During the entire follow-up period, 13 current/previous smokers died compared with only one non-smoker. Current/previous smoking status was associated with a significantly higher risk of dying both within 5 years and after the full follow-up period $(p=0.03$ and 0.01 , respectively) (Figure 3 ).

The risk of dying for male current/previous smokers over the entire follow-up period was significantly higher compared to the rest of the study population $(p<0.001)$.
A Ki67 index of $>2 \%$ was associated with a trend towards greater risk of dying within 5 years $(p=0.11)$. This association was significant for the full follow-up period $(p=0.04)$. Male gender combined with current/previous smoking status and Ki67 index $>2 \%$ was significantly associated with death within 5 years of follow-up compared with the rest of the study population $(p<0.0001)$.

Metastatic disease at diagnosis was a negative survival predictor both within 5 years of diagnosis and for the entire follow-up period (both $p<0.01$ ). No patient with nonmetastatic disease at diagnosis died within the first 5 years of follow-up (Figure 4).

The median $\mathrm{CgA}$ level was higher in patients who died, but the association was not significant $(p=0.22)$.

\section{Discussion}

In this retrospective study of patients with bronchial carcinoids from a Danish NET Centre, we observed a strong association between survival and current/previous smoking status. In accordance with previous studies, we found a higher mortality for patients with AC compared to patients 

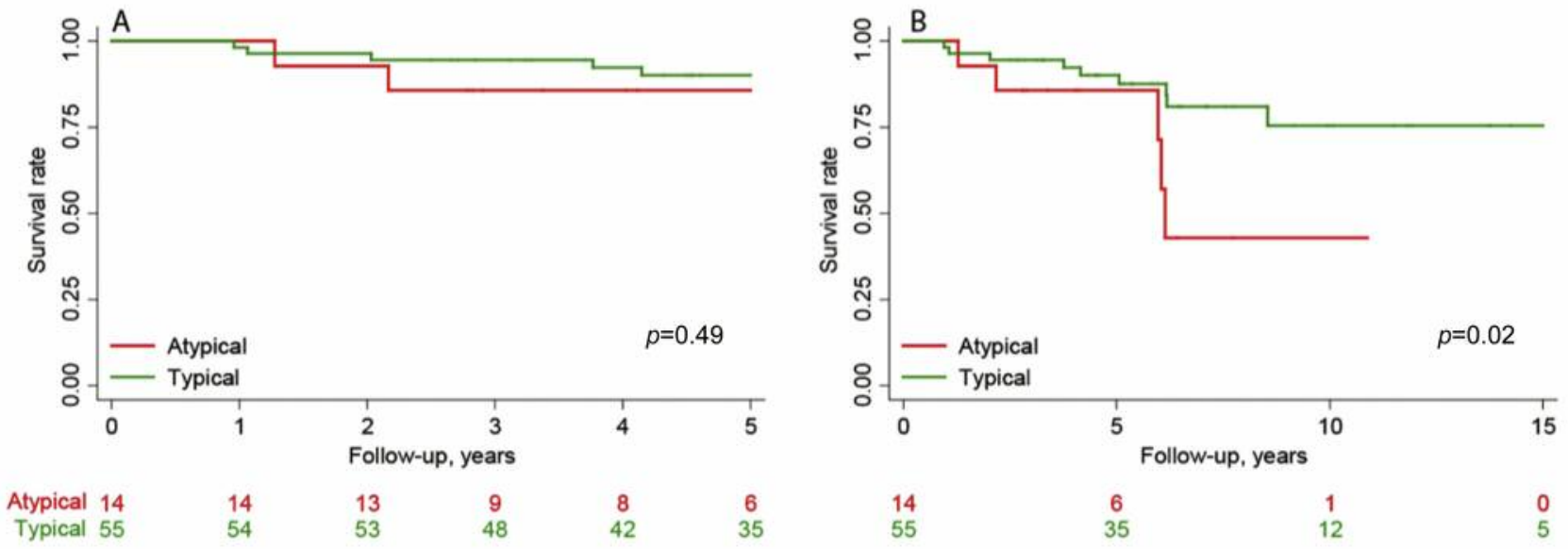

Figure 1. Survival rates for up to 5 years (A) and for full follow-up period (B) based on tumor type.
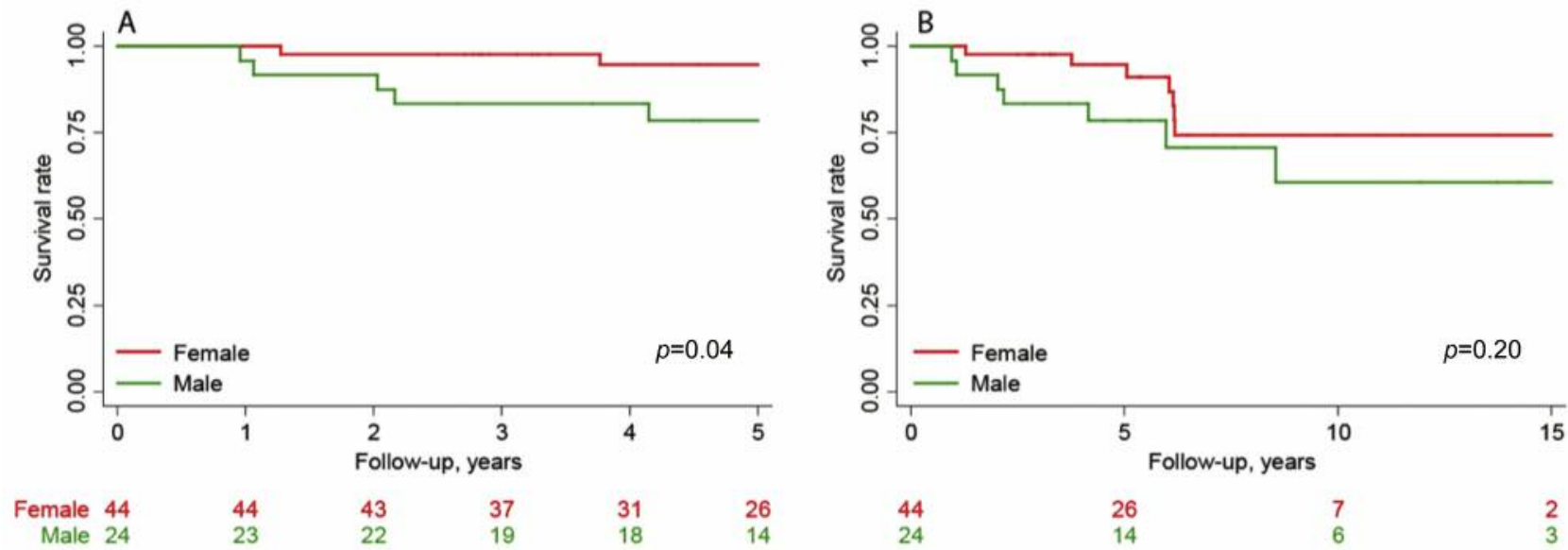

Figure 2. Survival rates for up to 5 years (A) and for full follow-up period (B) based on gender.

with TC and for patients with Ki67 index $>2 \%$. Metastatic disease at diagnosis and male gender were additional mortality predictors.

We did not demonstrate any difference in distribution of non-smokers and previous/current smokers in TC compared to AC. However, prevalence of current smokers in both TC and $\mathrm{AC}$ in our study $(60 \%$ and $69 \%, p=0.54)$ was considerably higher than prevalence in the general Danish population (approximately 20\% in 2013) (18). This may indicate that smoking is a risk factor for developing bronchial carcinoid. Several studies investigated this question, and results are conflicting. Patients with SCLC and LCNEC are frequently heavy-smokers. However, previous studies have been unable to demonstrate any clear association with the development of AC and TC $(1,12,13)$.
An Israeli study of 142 patients found the prevalence of smoking in the TC group to be almost that of the general population, whereas the prevalence was twice as high among patients with AC (8). Similarly, Froudarakis et al. described a higher prevalence of smoking, especially heavy smoking, in AC compared with TC patients (9). In our retrospective study of patients with bronchial carcinoid, we convincingly demonstrate that current and previous smoking status is associated with a poor survival. As far as we are aware, this has never been described previously. During the entire follow-up period, only one single patient died from the nonsmoker group compared with 13 in the current/previous smoker group. Smoking status thereby seems to be the strongest single predictor of mortality for patients with bronchial carcinoids. 

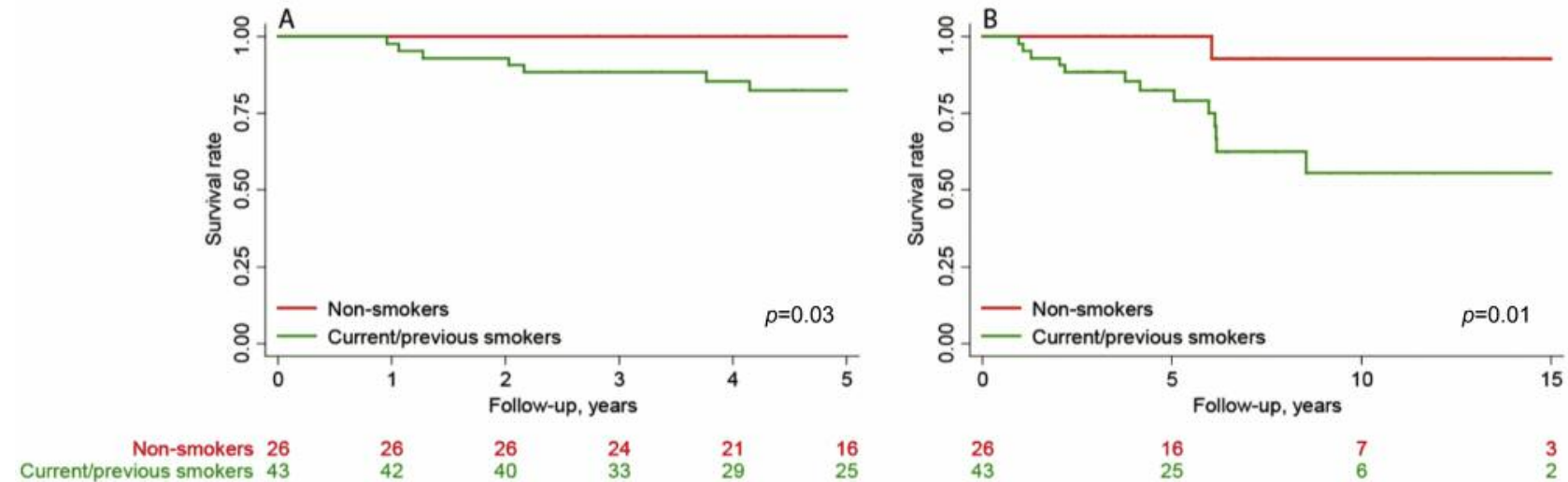

Figure 3. Survival rates for up to 5 years (A) and for full follow-up period (B) based on smoking status.
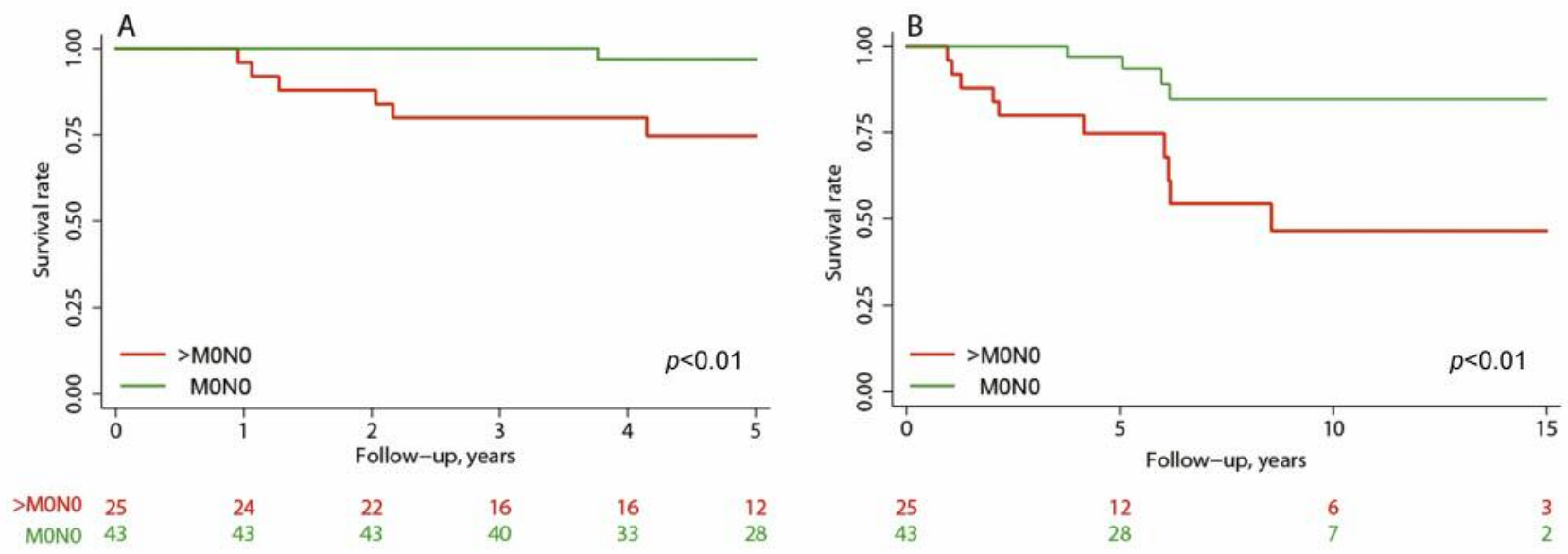

Figure 4. Survival rates for up to 5 years (A) and for full follow-up period (B) for metastatic (>MONO) versus non-metastatic (MONO) disease at diagnosis.

In the study of Froudarakis et al., histological type, stage at diagnosis, and the presence of distant metastases were significant predictors of mortality, whereas age, gender, smoking habits, tumor size, and lymph node involvement were not (9). Beasley et al. found that increased numbers of mitoses, tumor size $>3.5 \mathrm{~cm}$, and female gender were negative predictors of survival, whereas smoking was not (3). The same is found in several other studies regarding smoking $(12,13)$. In the present study, survival was associated with gender, Ki67-index, and metastatic disease at diagnosis. We found no difference in survival between TC and $\mathrm{AC}$ at five years of follow up. As found in previous studies, survival of AC patients was poorer looking at the entire follow-up period $(p<0.05)(1-3,5)$. Zahel et al. questioned the reliability of prognosis, when dividing patients according to diagnosis (TC/AC). They found a stronger association with prognosis when dividing patients according to the grading system for gastrointestinal neuroendocrine tumors (G1: $<2$ mitoses per $2 \mathrm{~mm}^{2}$ and/or Ki67-index $\leq 2$; G2: 2-20 mitoses per $2 \mathrm{~mm}^{2}$ and Ki-67-index between 3-20) (19). This supports our findings of Ki67-index being a stronger prognostic factor of survival than the TC/AC diagnosis.

Strengths of the present study are the number of wellcharacterized patients according to clinical, biochemical, and pathological features as well as imaging. Patients were followed in a highly specialized NET center. Patients had a similar distribution of age, gender, and tumor type compared with previous studies. $(4,8,9,13,20)$. Hence, they were representative of the population with bronchial carcinoids. 
Since all patients diagnosed with TC and AC from Western Denmark in the period 2000-2014 were referred to our NET Centre, we do not consider selection bias to be a problem.

However, our study has limitations. It is a retrospective study with a limited number of deaths, despite a relatively long follow-up period. This probably explains lack of difference in survival between $\mathrm{TC}$ and $\mathrm{AC}$ within the first five years. Analyzing the full follow-up period revealed a significantly poorer prognosis of patients with AC. However, numbers at risk later than five years of follow-up were very low, and data for the entire follow-up period should, therefore, be interpreted with caution.

In conclusion, we observed that current or previous smoking, metastatic disease at diagnosis and male gender are strong predictors of mortality in patients with bronchial carcinoids. Prospective studies with larger study populations and longer follow-up periods are required to further investigate these associations.

\section{Conflicts of Interest}

The Authors declare no conflicts of interest in regard to this study

\section{Acknowledgements}

Aarhus University Hospital ENETS Centre of Excellence has received research grants from Novartis and Ipsen; Henning Grønbæk has a clinical research grant from the NOVO Nordisk Foundation.

\section{References}

1 Gustafsson BI, Kidd M, Chan A, Malfertheiner MV and Modlin IM: Bronchopulmonary neuroendocrine tumors. Cancer 113: 521, 2008.

2 Travis WD: Advances in neuroendocrine lung tumors. Ann Oncol 21: vii65-71, 2010.

3 Beasley MB, Thunnissen FB, Brambilla E, Hasleton P, Steele R, Hammar SP, Colby TV, Sheppard M, Shimosato Y, Koss MN, Falk R and Travis WD: Pulmonary atypical carcinoid: predictors of survival in 106 cases. Hum Pathol 31: 1255-65, 2000.

4 Caplin ME, Baudin E, Ferolla P, Filosso P, Garcia-Yuste M, Lim E, Oberg K, Pelosi G, Perren A, Rossi RE, Travis WD and the ENETS consensus conference participants: Pulmonary neuroendocrine (carcinoid) tumors: European Neuroendocrine Tumor Society expert consensus and recommendations for best practice for typical and atypical pulmonary carcinoids. Ann Oncol 26: 1604-1620, 2015.

5 Skuladottir H, Hirsch FR, Hansen HH and Olsen JH: Pulmonary neuroendocrine tumors: Incidence and prognosis of histological subtypes. A population-based study in Denmark. Lung Cancer 37: 127-135, 2002.

6 Voortman J, Lee J-H, Killian JK, Suuriniemi M, Wang Y, Lucchi M, Smith WI, Meltzer P, Wang Y and Giaccone G: Array comparative genomic hybridization-based characterization of genetic alterations in pulmonary neuroendocrine tumors. Proc Natl Acad Sci USA 107: 13040-13045, 2010.
7 Johnen G, Krismann M, Jaworska M and Müller KM: CGHbefunde bei neuroendokrinen tumoren der lunge. Pathologe 24: 303-307, 2003.

8 Fink G, Krelbaum T, Yellin A, Bendayan D, Saute M, Glazer M and Kramer MR: Pulmonary carcinoid: Presentation, diagnosis, and outcome in 142 cases in Israel and review of 640 cases from the literature. Chest 119: 1647-1651, 2001.

9 Froudarakis M, Fournel P, Burgard G, Bouros D, Boucheron S, Siafakas NM and Emonot A: Bronchial carcinoids. A review of 22 cases. Oncology 53: 153-158, 1996.

10 Travis WD: Lung tumours with neuroendocrine differentiation. Eur J Cancer 45(Suppl 1): 251-66, 2009.

11 Hassan MM, Phan A, Li D, Dagohoy CG, Leary C and Yao JC: Risk factors associated with neuroendocrine tumors: A U.S.based case-control study. Int J Cancer 123: 867-873, 2008.

12 Harpole DH, Feldman JM, Buchanan S, Young WG and Wolfe WG: Bronchial carcinoid tumors: A retrospective analysis of 126 patients. Ann Thorac Surg 54: 50-55, 1992.

13 Cardillo G, Sera F, Di Martino M, Graziano P, Giunti R, Carbone L, Facciolo F and Martelli M: Bronchial carcinoid tumors: nodal status and long-term survival after resection. Ann Thorac Surg 77: 1781-5, 2004.

14 Hoej LB, Parkner T, Knudsen CS and Grønbaek H: A comparison of three chromogranin A assays in patients with neuroendocrine tumours. J Gastrointest Liver Dis 23: 419-424, 2014.

15 Brambilla E, Travis WD, Colby TV, Corrin B and Shimosato Y: The new World Health Organization classification of lung tumours. Eur Respir J 18: 1059-1068, 2001.

16 Pelosi G, Rindi G, Travis WD and Papotti M: Ki-67 Antigen in lung neuroendocrine tumors. J Thorac Oncol 9: 273-284, 2014.

17 UyBico SJ, Wu CC, Suh RD, Le NH, Brown K and Krishnam MS: Lung cancer staging essentials: the new TNM staging system and potential imaging pitfalls. Radiographics 30: 1163$81,2010$.

18 Christensen AI, Davidsen M, Ekholm O, Pedersen PV and Juel K: DANSKERNES SUNDHED - Den Nationale Sundhedsprofil 2013, 2014.

19 Zahel T, Krysa S, Herpel E, Stenzinger A, Goeppert B, Schirmacher P, Hoffmann H, Schnabel PA and Warth A: Phenotyping of pulmonary carcinoids and a Ki-67-based grading approach. Virchows Arch 460: 299-308, 2012.

20 Ferguson MK, Landreneau RJ, Hazelrigg SR, Altorki NK, Naunheim KS, Zwischenberger JB, Kent M and Yim AP: Longterm outcome after resection for bronchial carcinoid tumors. Eur J Cardiothorac Surg 18: 156-161, 2000.

21 Reed N, Couvelard A and Ruszniewski P: 12th Annual ENETS Conference for the Diagnosis and Treatment of Neuroendocrine Tumor Disease. March 11-13, 2015, Barcelona, Spain: Abstracts. Neuroendocrinology: 77-168, 2015. 